\title{
Violência contra o idoso no Rio Grande do Sul, Brasil: análise das notificações de 2009 a 2016*
}

Violence against elderly in Rio Grande do Sul, Brazil: analysis of notifications from 2009 to 2016

Violencia contra los ancianos en Rio Grande do Sul, Brasil: Análisis de las notificaciones del 2009 al 2016

Guilherme Tavares de Arruda

Sheila Kocourek Jairo da Luz Oliveira

RESUMO: Estudo descritivo e documental realizado por meio de uma busca on-line no Sinan-Net, com o objetivo de analisar as notificações de violência contra o idoso, no Rio Grande do Sul, no período de 2009 a 2016. Observou-se um aumento crescente de notificações de violência contra o idoso no estado, principalmente entre mulheres e brancos. Os casos de violência, em sua maioria, ocorreram em meio urbano, com maior frequência de violência tipo física e psicológica, por força corporal e ameaça.

Palavras-chave: Idoso; Violência; Notificação.

\footnotetext{
* O estudo foi desenvolvido na Universidade Federal de Santa Maria, no curso de Graduação em Serviço Social do Centro de Ciências Sociais e Humanas da Universidade Federal de Santa Maria (UFSM), Santa Maria, Rio Grande do Sul, Brasil. Núcleo de Estudo, Pesquisa e Extensão em Gerontologia, Serviço Social e Saúde (NEPEGSSS).

Arruda, G. T. de, Kocourek, S., \& Oliveira, J. da L. (2018). Violência contra o idoso no Rio Grande do Sul, Brasil: análise das notificações de 2009 a 2016. Revista Kairós-Gerontologia, 21(3), 181-192. ISSNe 2176-901X.

São Paulo (SP), Brasil: FACHS/NEPE/PEPGG/PUC-SP
} 
ABSTRACT: A descriptive and documentary study was carried out by means of an online search on Sinan-Net, with the objective of analyzing the reports of violence against the elderly, in Rio Grande do Sul, from 2009 to 2016. There was a growing increase of notifications of violence against the elderly in the state, especially among women and whites. Most cases of violence occurred in an urban environment, with a greater frequency of physical and psychological violence, by corporal force and threat.

Keywords: Aged; Violence; Notification.

RESUMEN: Estudio descriptivo y documental realizado a través de una búsqueda en línea en Sinan-net, con el objetivo de analizar los informes de violencia contra los ancianos en Rio Grande do Sul, del 2009 al 2016. Hubo un creciente aumento en los informes de violencia contra los ancianos en el estado, especialmente entre las mujeres y los blancos. La mayoría de los casos de violencia ocurrieron en zonas urbanas, con una mayor frecuencia de violencia física y psicológica, por fuerza corporal y amenaza.

Palabras clave: Ancianos; Violencia; Notificación.

\section{Introdução}

O envelhecimento populacional acelerado é uma realidade em diversos países. Segundo dados do Instituto Brasileiro de Geografia e Estatística (2010), estima-se que em 2025 o número de indivíduos com mais de 60 anos de idade quase triplicará no Brasil, tornando-o o sexto país com maior número de idosos no mundo (Veras, 2009).

No Rio Grande do Sul (RS), o envelhecimento acelerado ocorre de modo diferenciado ao do restante do Brasil, em decorrência de o RS ser um dos estados com maior índice de desenvolvimento humano e possuir maior expectativa de vida e de idosos na população (Goottlieb, Schwanke, Gomes, \& Da Cruz, 2011). Entretanto, o processo de envelhecimento leva à ocorrência de patologias crônico-degenerativas que tornam o idoso mais frágil e suscetível a maus-tratos e à violência (Saad, 2011).

Segundo Machado e Queiroz (2002), os maus-tratos contra a pessoa idosa podem ser definidos como atos ou omissões que produzem dano ou perigo para a saúde ou bem-estar do indivíduo, bem como dano físico ou mental, abuso sexual, negação de alimentos, de vestuário e de assistência médica. 
Existem diversas formas de violência contra o idoso, dentre elas a violência física, a sexual, a psicológica ou moral, a financeira, entre outras, que trazem consequências de âmbito biopsicossocial no indivíduo e vão desde o surgimento de lesões, alterações do estado psicológico, medo e até a morte (Aguiar, Leite, Dias, Mattos, \& Lima, 2015; Bellal, et al., 2015).

Em 2009, o número de óbitos de idosos no Brasil totalizou 21.453 mortes, sendo 1.929 destas em decorrência de maus-tratos e violência (Mascarenhas, et al., 2011).

De 2009 para os dias atuais, esses números têm aumentado drasticamente, tornando um problema de saúde pública (Correia, Leal, Marques, Salgado, \& Melo, 2012). Diante disso, conforme a Lei n. ${ }^{o}$ 12.461/2011, "os casos de suspeita ou confirmação de violência praticada contra idosos serão objeto de notificação compulsória pelos serviços de saúde públicos e privados à autoridade sanitária, bem como serão obrigatoriamente comunicados por eles" (Brasil, 2011). Para tal, a notificação serve como um recurso formal de comunicação assistencial a fim de que ações sejam criadas para o enfrentamento da violência em determinados locais e necessidades (Assis, Avanci, Pesce, Pires, \& Gomes, 2012).

Em 2009, o Ministério da Saúde implantou o Sistema de Informação de Agravos de Notificação (Sinan) como forma de garantir a proteção à vítima, com amparo legal aos profissionais da saúde, de modo a impulsionar os registros de violência no Brasil e diminuir os casos de sua omissão. Anteriormente ao Sinan, as notificações sobre violência de cada município eram registradas em fichas próprias; isso gerava informações incompletas e heterogêneas. Nesse atual sistema, é possível identificar a dimensão da violência no país e o perfil das notificações, além de padronizar as informações oriundas de cada município (Assis, Avanci, Pesce, Pires, \& Gomes, 2012).

Diversos artigos analisam as notificações de violência contra o idoso em cidades de médio a grande porte do Brasil, mas a literatura ainda carece de estudos que tratem dessa temática no RS (Irigaray, Esteves, Pacheco, Grassi-Oliveira, \& Argimoni, 2016; Santana, Vasconcelos, \& Coutinho, 2016). Além disso, a subnotificação ainda é uma realidade e pode ser explicada pela dificuldade em se denunciar a violência doméstica contra os idosos, pela falta de capacitação dos profissionais de saúde em detectar o problema e pela insegurança da vítima em prestar queixa formal (Mascarenhas, et al., 2011). Diante disso, e em decorrência da necessidade de se conhecer o perfil das notificações no RS, este estudo tem como objetivo analisar as notificações de violência contra o idoso no RS no período de 2009 a 2016. 


\section{Métodos}

Trata-se de um estudo descritivo e documental realizado por meio de uma busca on-line no banco de dados do Sinan-Net: investigação de violência doméstica, sexual e/ou outras violências (Rio Grande do Sul, 2017). A busca foi realizada entre os meses de agosto a setembro de 2017 e categorizada conforme os seguintes tipos de violência ocorridos entre os anos de 2009 e 2016 no RS: lesão autoprovocada, violência física, violência psicológica ou moral, violência por negligência ou abandono, violência por tortura, violência sexual, violência financeira ou econômica e outro tipo de violência.

Para a busca, foram utilizadas as seguintes variáveis, conforme ano e tipo de violência: sexo das vítimas, cor da pele/etnia das vítimas, zona de ocorrência e forma de agressão.

Foram incluídos na busca dados referentes à faixa etária/ciclo de vida de indivíduos com 60 anos de idade ou mais. Os dados foram analisados por meio de estatística descritiva a apresentados por meio de gráfico e tabela.

\section{Resultados}

No RS, o número de notificações de violência contra o idoso aumentou de 25 casos, em 2009, para 2.402 casos, em 2016, totalizando 12.439 registros entre o período analisado, conforme Figura 1.

Figura 1 - Distribuição do número de notificações de violência contra o idoso no RS, no período de 2009 a 2016

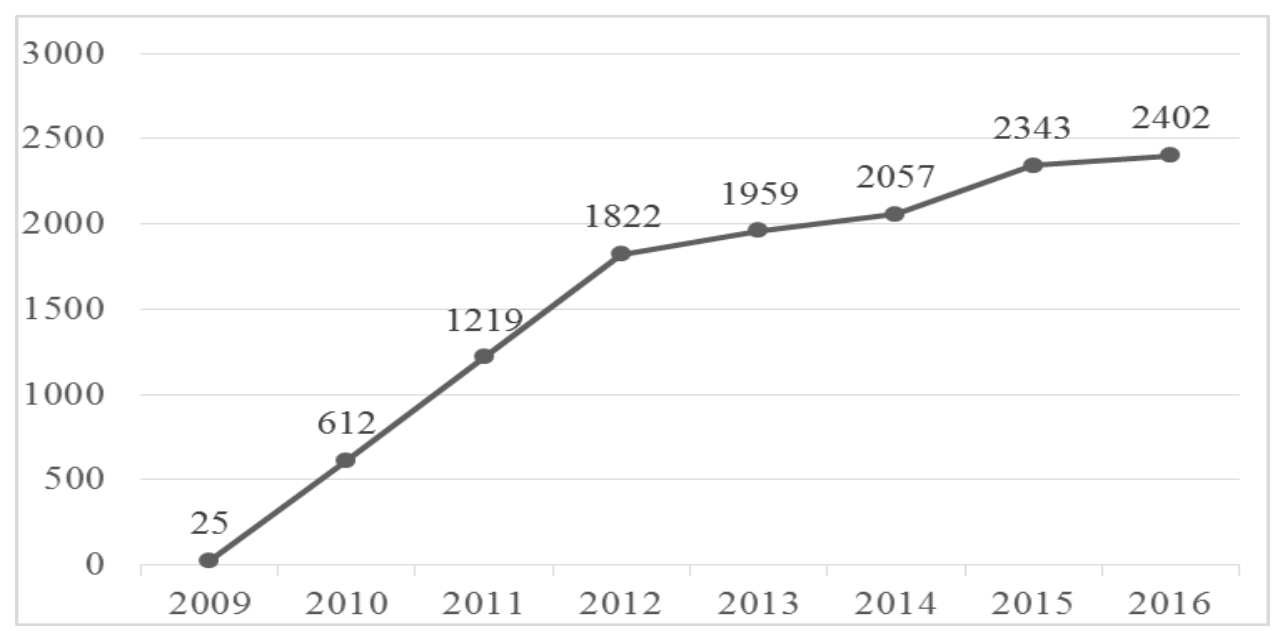

Fonte: Sinan/TabNet-RS (2009-2016)

Arruda, G. T. de, Kocourek, S., \& Oliveira, J. da L. (2018). Violência contra o idoso no Rio Grande do Sul, Brasil: análise das notificações de 2009 a 2016. Revista Kairós-Gerontologia, 21(3), 181-192. ISSNe 2176-901X.

São Paulo (SP), Brasil: FACHS/NEPE/PEPGG/PUC-SP 
A distribuição do número e proporção das notificações de violência contra o idoso no RS, no período entre 2009 a 2016, segundo sexo da vítima, cor da pele/etnia da vítima, tipo de violência, zona de ocorrência e forma de agressão estão apresentados na Tabela 1 acima.

Em relação ao sexo e à cor da pele/etnia das vítimas, a maioria é composta por mulheres $(59,8 \%)$ e brancos $(83,5 \%)$. Entre o período analisado, houve maior número de notificações sobre violência física $(34,4 \%)$, seguido por violência psicológica ou moral $(22,2 \%)$; violência por negligência ou abandono (18,2\%); lesão autoprovocada (11\%); outro tipo de violência $(5,7 \%)$; violência financeira ou econômica $(5,6 \%)$; violência por tortura (1,9\%); e violência sexual (1\%). Em geral, observa-se um aumento crescente no número de notificações em cada tipo de violência com o passar dos anos.

A zona de maior ocorrência de violência foi a urbana, totalizando 76,9\% dos casos; e as formas de agressão mais frequentes ocorreram por força corporal/espancamento $(n=4956)$ e ameaça $(n=3478)$.

Tabela 1 - Distribuição do número e proporção das notificações de violência contra o idoso no RS, no período entre 2009 a 2016, segundo sexo da vítima, tipo de violência, cor da pele/etnia da vítima, zona de ocorrência e forma de agressão

\begin{tabular}{|c|c|c|c|c|c|c|c|c|c|}
\hline & 2009 & 2010 & 2011 & 2012 & 2013 & 2014 & 2015 & 2016 & $\begin{array}{l}\text { Total } \\
\text { n }(\%)\end{array}$ \\
\hline \multicolumn{10}{|l|}{ Sexo da vítima } \\
\hline Feminino & 20 & 323 & 705 & 1121 & 1185 & 1261 & 1370 & 1463 & $7448(59,8 \%)$ \\
\hline Masculino & 5 & 288 & 514 & 701 & 774 & 796 & 973 & 939 & $4990(40,1 \%)$ \\
\hline Não informado & 0 & 1 & 0 & 0 & 0 & 0 & 0 & 0 & $1(0,1 \%)$ \\
\hline \multicolumn{10}{|c|}{ Cor da pele/etnia da vítima } \\
\hline Branca & 15 & 519 & 1013 & 1488 & 1654 & 1697 & 1970 & 2029 & $10385(83,5 \%)$ \\
\hline Parda & 5 & 32 & 99 & 143 & 149 & 178 & 154 & 155 & $915(7,3 \%)$ \\
\hline Preta & 5 & 33 & 61 & 110 & 71 & 106 & 95 & 101 & $582(4,7 \%)$ \\
\hline Não informado & 0 & 27 & 45 & 76 & 70 & 63 & 117 & 110 & $508(4,1 \%)$ \\
\hline Indígena & 0 & 0 & 1 & 3 & 5 & 10 & 3 & 5 & $27(0,2 \%)$ \\
\hline Amarela & 0 & 1 & 0 & 2 & 10 & 3 & 4 & 2 & $22(0,2 \%)$ \\
\hline \multicolumn{10}{|l|}{ Tipo de violência } \\
\hline Física & 8 & 255 & 454 & 628 & 662 & 686 & 746 & 837 & $4276(34,4 \%)$ \\
\hline Psicológica ou moral & 8 & 102 & 227 & 404 & 452 & 472 & 527 & 570 & $2762(22,2 \%)$ \\
\hline
\end{tabular}




\begin{tabular}{|c|c|c|c|c|c|c|c|c|c|}
\hline Por negligência/abandono & 9 & 117 & 234 & 296 & 398 & 382 & 469 & 363 & $2268(18,2 \%)$ \\
\hline Lesão autoprovocada & 0 & 57 & 129 & 203 & 199 & 228 & 269 & 289 & $1374(11 \%)$ \\
\hline Outro tipo & 0 & 28 & 74 & 117 & 79 & 113 & 145 & 148 & $704(5,7 \%)$ \\
\hline Financeira ou econômica & 0 & 29 & 63 & 114 & 122 & 120 & 125 & 122 & $695(5,6 \%)$ \\
\hline Por tortura & 0 & 13 & 26 & 46 & 34 & 39 & 35 & 43 & $236(1,9 \%)$ \\
\hline Sexual & 0 & 11 & 12 & 14 & 13 & 17 & 27 & 30 & $124(1 \%)$ \\
\hline \multicolumn{10}{|l|}{ Zona de ocorrência } \\
\hline Urbano & 25 & 464 & 932 & 1419 & 1489 & 1544 & 1794 & 1904 & $9571(76,9 \%)$ \\
\hline Rural & 0 & 123 & 222 & 334 & 335 & 378 & 343 & 347 & $2082(16,8 \%)$ \\
\hline Não informado & 0 & 22 & 51 & 43 & 105 & 113 & 165 & 137 & $636(5,1 \%)$ \\
\hline Periurbano & 0 & 3 & 14 & 26 & 30 & 22 & 41 & 14 & $150(1,2 \%)$ \\
\hline \multicolumn{10}{|l|}{ Forma de agressão* } \\
\hline Força corporal/espancamento & 12 & 233 & 476 & 746 & 819 & 822 & 876 & 972 & 4956 \\
\hline Ameaça & 0 & 112 & 233 & 571 & 592 & 578 & 738 & 654 & 3478 \\
\hline Outra & 3 & 125 & 283 & 320 & 353 & 447 & 655 & 665 & 2851 \\
\hline Enforcamento & 0 & 56 & 119 & 213 & 208 & 206 & 260 & 242 & 1304 \\
\hline Objeto perfuro-cortante & 6 & 55 & 80 & 123 & 133 & 134 & 195 & 193 & 919 \\
\hline Objeto contundente & 2 & 25 & 53 & 88 & 92 & 118 & 117 & 106 & 601 \\
\hline Arma de fogo & 0 & 43 & 48 & 100 & 78 & 61 & 88 & 90 & 508 \\
\hline Envenenamento & 0 & 13 & 29 & 44 & 48 & 89 & 72 & 106 & 401 \\
\hline Substância/objeto quente & 0 & 1 & 14 & 12 & 12 & 12 & 27 & 25 & 103 \\
\hline
\end{tabular}

Fonte: Sinan/TabNet-RS (2009-2016)

*Nesta variável, mais de uma forma de agressão poderia ocorrer.

\section{Discussão}

A violência e os maus-tratos contra os idosos têm sido tema de muito interesse e discussão no meio científico. Entretanto, a literatura carece de estudos sobre essa temática no RS (Irigaray, Esteves, Pacheco, Grassi-Oliveira, \& Argimoni, 2016; Santana, Vasconcelos, \& Coutinho, 2016).

Em nosso estudo, houve um aumento crescente das notificações no período analisado, totalizando 12.439 registros de violência no estado. $\mathrm{O}$ ano de 2016 teve o maior número, com 19,3\% do total de registros. 
Tal fato pode ser devido ao crescente incentivo de políticas públicas pela notificação da violência, visto que a subnotificação vem diminuindo com o passar dos anos, como demonstrado no presente estudo.

No entanto, é preciso ressaltar que, muitas vezes, as vítimas não denunciam seus agressores por medo ou por serem dependentes deles; nesse caso, quando o agressor é algum familiar ou cuidador (Irigaray, Esteves, Pacheco, Grassi-Oliveira, \& Argimoni, 2016).

Além disso, o número de notificações poderia ser ainda maior caso houvesse um sistema de dados, como o Sinan, anterior a 2009, e profissionais qualificados para o manuseio desses dados (Pinto, \& Assis, 2015).

Em relação ao sexo e à cor da pele/etnia das vítimas, a maioria destas eram do sexo feminino e brancas. Dados semelhantes são evidenciados em diversos estudos (Rodrigues, et al., 2017; Garbin, Joaquim, Rovida, \& Garbin, 2016; Grilo, \& Lombardi Júnior, 2015).

A prevalência de vítimas de cor de pele branca encontrada neste estudo corrobora com o atual perfil étnico do RS, explicado pelo aumento do fluxo de imigrantes europeus para o Sul do Brasil durante a época colonial (Santos, 2017).

Sobre o sexo das vítimas, a literatura aponta a existência de uma relação de gênero com a violência, na qual a mulher é a principal vítima (Paiva, \& Tavares, 2015; Garbin, Joaquim, Rovida, \& Garbin, 2016).

Para Garbin, Joaquim, Rovida, \& Garbin (2016), tal fato é consequência de uma sociedade machista e violenta onde, historicamente, a mulher apresentava dependência ao homem. Outros autores (Paiva, \& Tavares, 2015; Santana, Vasconcelos, \& Coutinho, 2016) relatam que, ainda hoje, vive-se o pensamento hierárquico entre homens e mulheres, em que as mulheres são discriminadas e inferiorizadas, o que justificaria a alta prevalência de violência contra o sexo feminino.

Dentre o período analisado neste estudo, a violência do tipo física $(34,4 \%)$ foi a mais frequente, seguida pela violência psicológica ou moral $(22,2 \%)$ e por negligência/abandono $(18,2 \%)$.

Esses números mostram-se quase igualmente distribuídos desde 2009, pois apenas em 2011 as notificações de negligência/abandono ultrapassaram as de violência psicológica ou moral. 
Segundo Sampaio, Ferreira, Sampaio, Souza, Prado, \& Reis (2017), o próprio envelhecimento e suas consequências são fatores que contribuem para a alta prevalência de violência física contra os idosos pois, com o envelhecimento, o idoso torna-se mais dependente fisicamente e, consequentemente, mais vulnerável a maus-tratos (Reis, Gomes, Reis, Menezes, \& Carneiro, 2014).

Alguns estudos mostram a violência física como o principal tipo de violência contra o idoso (Gil, Santos, Kislaya, Santos, Mascoli, Ferreira, \& Vieira, 2015; Sampaio, Ferreira, Sampaio, Souza, Prado, \& Reis, 2017). Entretanto, em estudo de Irigaray, Esteves, Pacheco, Grassi-Oliveira, \& Argimoni (2016), o abuso psicológico foi o mais frequente. Para os autores, esse tipo de violência ocorre por ameaça de abandono do agressor e como forma de manter o idoso submisso. Sabe-se que tal violência pode gerar consequências graves à saúde da vítima, levando à depressão, sentimento de desvalorização e outros transtornos psicológicos que acabam interferindo negativamente sobre sua qualidade de vida e bem-estar social.

Em relação à zona de ocorrência, a maioria das notificações de violência deste estudo ocorreram em zonas urbanas (76,9\%). Apesar de a violência urbana ser pouca explorada na literatura (Santana, Vasconcelos, \& Coutinho, 2016), principalmente em relação ao idoso, é necessário identificar suas causas, devido à elevada frequência em nosso estudo.

Silva e Dias (2016) relacionam a maior ocorrência de violência em meio urbano com o ambiente local, além de estarem envolvidas questões sociais, como o abuso de drogas, baixa renda e ausência de suporte social. Isso faz da violência um problema de saúde pública complexo, que necessita de maior articulação da sociedade e da política para a implementação e implantação de políticas que atendam às necessidades da população e que compreendam o processo do envelhecimento junto à cidade.

Quanto à forma de agressão, força corporal/espancamento e ameaça foram as mais frequentes. Vale destacar que, durante o registro de violência, a agressão pode ocorrer por mais de uma forma, justificando a falta de proporção nesses dados em relação ao número total de notificações. Resultados semelhantes foram encontrados por outro estudo (Sampaio, Ferreira, Sampaio, Souza, Prado, \& Reis, 2017).

O aumento do número de notificações dessas formas de agressão em idosos pode estar relacionada à violência física e psicológica ocorridas, pois o indivíduo sofre ameaças antes de ser agredido fisicamente (Paiva, \& Tavares, 2015). 
Além disso, idosos mais frágeis, que possuem déficits cognitivos, incontinência e dependência para locomoção também são mais propensos a agressões devido à sobrecarga física e psicológica imposta ao cuidador ou familiar (Grilo, \& Lombardi Júnior, 2015).

Com o aumento da população de idosos no Brasil e no RS, o número de notificações de violência contra indivíduos dessa faixa etária também tende a aumentar. Estudos como este tornam-se necessários para se conhecer o perfil das violências em nosso estado e, assim, promover estratégias de prevenção e intervenção para este problema. Além disso, também é fundamental a discussão desse tema entre os profissionais da saúde para a promoção de melhor instrumentalização da ficha de notificação.

Como limitações do estudo, salienta-se a falta de pesquisas atuais encontradas na literatura, sobre violência no RS (Irigaray, Esteves, Pacheco, Grassi-Oliveira, \& Argimoni, 2016), que possam servir para maiores comparações com os resultados deste estudo e uma análise maior sobre o perfil das vítimas e agressores. Assim, sugere-se a realização de novas pesquisas que busquem mais informações junto às delegacias e vítimas de violência, e não somente a sistemas de dados.

\section{Conclusão}

Observou-se um aumento crescente, entre o período analisado, de notificações de violência contra o idoso no RS, principalmente entre mulheres e brancos. Também se constatou que os casos de violência, em sua maioria, ocorreram em meio urbano, sendo as violências, dos tipos física e psicológica ou moral, as mais frequentes, juntamente às formas força corporal/espancamento e ameaça.

Visa-se, assim, a contribuir para o desenvolvimento de políticas públicas para o enfrentamento da violência contra o idoso no estado, bem como identificar o perfil das vítimas, locais de maior ocorrência, tipos e formas de violência mais frequentes. Desse modo, buscando-se promover estratégias específicas para cada um desses dados encontrados. 


\section{Referências}

Aguiar, M. P. C., Leite, H. A., Dias, I. M., Mattos, M. C. T., \& Lima, W. R. (2015). Violência contra idosos: descrição de casos no Município de Aracaju, Sergipe, Brasil. Escola Anna Nery Revista de Enfermagem, 19(2), 343-349. Recuperado em 01 dezembro, 2017, de: http://www.scielo.br/pdf/ean/v19n2/1414-8145-ean-19-02-0343.pdf.

Assis, S. G., Avanci, J. Q., Pesce, R. P., Pires, T. O., \& Gomes, D. L. (2012). Notificações de violência doméstica, sexual e outras violências contra crianças no Brasil. Ciência \& Saúde Coletiva, 17(9), 2305-2317. Recuperado em 01 dezembro, 2017, de: http://www.scielo.br/pdf/csc/v17n9/a12v17n9.pdf.

Bellal, J., Khalil, M., Zangbar, B., Kulvatunyou, N., Orouji, T., Pandit, V., Okeeffe, T. S., Tang, A., Gries, L., Friese, R. S., Rhee, P. M., \& Davis, J. W. (2015). Prevalence of Domestic Violence Among Trauma Patients. JAMA Surgery, 150(12), 1177-1183. Recuperado em 01 dezembro, 2017, de: https://doi.org/10.1001/jamasurg.2015.2386.

Brasil. (2011). Lei n. ${ }^{\circ} 12.461$, de 26 de julho de 2011. Altera a Lei n. ${ }^{\circ} 10.741$, de $1^{\circ}$ de outubro de 2003, para estabelecer a notificação compulsória dos atos de violência praticados contra o idoso atendido em serviço de saúde. Diário Oficial da União.

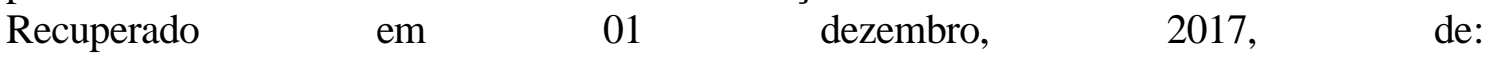
https://resenhasdeleispr.blogspot.com/2011/07/resenhas-lei-n-12461-de-2672011-e.html.

Carvalho, J. A. M., \& Rodríguez-Wong, L. L. (2008). A transição da estrutura etária brasileira na primeira metade do século XXI. Cadernos de Saúde Pública, 24(3), 597-605. Recuperado em 01 dezembro, 2017, de: http://www.scielo.br/pdf/csp/v24n3/13.pdf.

Correia, T. M. P., Leal, M. C. C., Marques, A. P. O., Salgado, R. A. G., \& Melo, H. M. A. (2012). Profile of elderly in violence situation assisted at an emergency service in Recife-PE. Revista Brasileira de Geriatria e Gerontologia, 15(3), 529-536. Recuperado em 01 dezembro, 2017, de: http://www.scielo.br/pdf/rbgg/v15n3/v15n3a13.pdf.

Garbin, C. A. S., Joaquim, R. C., Rovida, T. A. S., \& Garbin, A. J. I. (2016). Elderly victims of abuse: a five year document analysis. Revista Brasileira de Geriatria e Gerontologia, 19(1), 87-94. Recuperado em 01 dezembro, 2017, de: http://dx.doi.org/10.1590/1809-9823.2016.15037.

Gil, A. P., Santos, A. J., Kislaya, I., Santos, C., Mascoli, L., Ferreira, A. I., \& Vieira, D. N. (2015). Estudo sobre pessoas idosas vítimas de violência em Portugal: sociografia da ocorrência. Cadernos de Saúde Pública, 31(6), 1234-1246. Recuperado em 01 dezembro, 2017, de: https://doi.org/10.1590/0102-311X00084614.

Goottlieb, M. G. V., Schwanke, C. H. A., Gomes, I., \& Da Cruz, I. B. M. (2011). Envelhecimento e longevidade no Rio Grande do Sul: um perfil histórico, étnico e de morbimortalidade dos idosos. Revista Brasileira de Geriatria e Gerontologia, 12(2), 365-380. Recuperado em 01 dezembro, 2017, de: http://www.scielo.br/pdf/rbgg/v14n2/v14n2a16.

Grilo, P. M. S., \& Lombardi Júnior, I. (2015). Maus-tratos a idosos: perfil das vítimas, vínculo com o agressor e atuação dos profissionais. Estudos Interdisciplinares sobre Envelhecimento, 20(2), 611-624. Recuperado em 01 dezembro, 2017, de: https://seer.ufrgs.br/RevEnvelhecer/article/view/50955. 
Instituto Brasileiro de Geografia e Estatística. (2015). Síntese de Indicadores Sociais: uma análise das condições de vida da população brasileira. Recuperado em 01 dezembro, 2017, de: https://biblioteca.ibge.gov.br/visualizacao/livros/liv95011.pdf.

Irigaray, T. Q., Esteves, C. S., Pacheco, J. T. B., Grassi-Oliveira, R., \& Argimoni, I. I. L. (2016). Maus-tratos contra idosos em Porto Alegre, Rio Grande do Sul: um estudo documental. Estudos de Psicologia (Campinas), 33(3), 543-551. Recuperado em 01 dezembro, 2017, de: doi: 10.1590/1982-02752016000300017.

Machado, L., \& Queiroz, Z. V. (2002). Negligência e Maus-Tratos. In: Freitas, E. V., et al. Tratado de Geriatria e Gerontologia. Rio de Janeiro, RJ: Guanabara Koogan.

Mascarenhas, M. D. M., Monteiro, R. A., Sá, N. N. B. de., Gonzaga, L. A. A., Neves, Silva, M. M. A., \& Malta, D. C. (2011). Epidemiologia das causas externas no Brasil: mortalidade por acidentes e violências no período de 2000 a 2009. In: Brasil. Ministério da Saúde (MS). Secretaria de Vigilância em Saúde. Saúde Brasil 2010: uma análise da situação de saúde e de evidências selecionadas de impacto de ações de vigilância em saúde. Brasília, DF: MS.

Paiva, M. M., \& Tavares, D. M. S. (2015). Violência física e psicológica contra idosos: prevalência e fatores associados. Revista Brasileira de Enfermagem, 68(6), 727-733 Recuperado em 01 dezembro, 2017, de: doi: http://dx.doi.org/10.1590/00347167.2015680606i.

Pinto, L. W., \& Assis, S. G. (2015). Estudo descritivo das tentativas de suicídio na população idosa brasileira, 2000-2014. Ciência e Saúde Coletiva, 20(6), 1681-1692. Recuperado em 01 dezembro, 2017, de: doi: 10.1590/1413-81232015206.03532015.

Reis, L. A., Gomes, N. P., Reis, L. A., Menezes, T. M. O., \& Carneiro, J. B. (2014). Expressão da violência intrafamiliar contra idosos. Acta Paulista de Enfermagem, 27(5), 434-439. Recuperado em 01 dezembro, 2017, de: doi: 10.1590/1982-0194201400072.

Rio Grande do Sul. (2017). Secretaria Estadual da Saúde. Tabnet: Dados epidemiológicos - SINAN: violência doméstica, sexual e/ou outras, RS, 2009-2016. Santa Maria, RS: o autor. Recuperado em: http://200.198.173.165/scripts/deftohtm.exe?snet/violencianet.

Rodrigues, R. A. P., Monteiro, E. A., Santos, A. M. R. dos, Pontes, M. de L. de F., Fhon, J. R. S., Bolina, A. F., Seredynskyj, F. L., Almeida, V. C., Giacomini, S. B. L., Defina, G. P. C., \& Silva, L. M. (2017). Violência contra idosos em três municípios brasileiros. Revista Brasileira de Enfermagem, 70(4), 816-824. Recuperado em 01 dezembro, 2017, de: http://dx.doi.org/10.1590/0034-7167-2017-0114.

Saad, P. M. (2011). Demographic trends in Latin America and the Caribbean. In: Cotlear, D. (Editor). Population aging: is Latin America ready?, 43-77. Recuperado em 01 dezembro, 2017, de: http://documents.worldbank.org/curated/en/ 117291468299147625/pdf/588420PUB0Popu11public10BOX353816B0.pdf.

Sampaio, L. S., Ferreira, M. J. S., Sampaio, T. S. O., Souza, W. P., Prado, A. P. S., \& Reis, L. A. (2017). Violência física em idosos. C\&D-Revista Eletrônica da FAINOR, 10(2), 188-200. Recuperado em 01 dezembro, 2017, de: file://C:/Users/Dados/Downloads/661-2467-1-PB.pdf. 
Santana, I. O., Vasconcelos, D. C., \& Coutinho, M. P. L. (2016). Prevalência da violência contra o idoso no Brasil: revisão analítica. Arquivos Brasileiros de Psicologia, 68(1), 126-139. Recuperado em 01 dezembro, 2017, de: http://seer.psicologia.ufrj.br/index.php/abp/article/view/931/997.

Santos, M. O. (2017). Reescrevendo a história: imigrantes italianos, colonos alemães, portugueses e a população brasileira no sul do Brasil. Revista Tempo e Argumento, 9(20), 230-246. Recuperado em 01 dezembro, 2017, de: doi: $10.5965 / 2175180309202017230$.

Silva, C. F. S., \& Dias, C. M. S. B. (2016). Violência Contra Idosos na Família: Motivações, Sentimentos e Necessidades do Agressor. Psicologia: Ciência e Profissão, 36(3), 637-652. Recuperado em 01 dezembro, 2017, de: doi: 10.1590/19823703001462014.

Veras, R. (2009). Envelhecimento populacional contemporâneo: demandas, desafios e inovações. Revista de Saúde Pública, 43(3), 548-554. Recuperado em 01 dezembro, 2017, de: http://dx.doi.org/10.1590/S0034-89102009005000025.

Recebido em 03/01/2018

Aceito em 30/06/2018

Guilherme Tavares de Arruda - Acadêmico do Curso de Fisioterapia, Universidade

Federal de Santa Maria, Curso de Fisioterapia, UFSM. Santa Maria, RS, Brasil.

E-mail: gui_tavares007@hotmail.com

Sheila Kocourek - Professora Adjunta do Departamento de Serviço Social, Universidade Federal de Santa Maria (UFSM), Santa Maria, RS.

E-mail: sheilakocourek@gmail.com

Jairo da Luz Oliveira - Professor Adjunto do Departamento de Serviço Social, Universidade Federal de Santa Maria (UFSM), Santa Maria, RS.

E-mail: jairooliveira.ufsm@gmail.com 